\title{
Video Advance Directives: A Turning Point for Advance Decision-Making? A Consideration of Their Roles and Implications for Law and Practice
}

\author{
Hui Yun Chan ${ }^{1}$ \\ Published online: 5 October 2019 \\ (c) The Author(s) 2019
}

\begin{abstract}
Questions regarding making and implementing care preferences through advance directives have become increasingly significant as the greying population grows with rising numbers of people experiencing incapacity. Currently, there is no consensus in the format for making advance directives. Recent developments highlighted the use of recording technology as an option to counter the challenges of written forms. Services offering video and audio recording available for online and offline storage are emerging in the United States. These services presumably strengthen a person's expression of care preferences for healthcare providers in making treatment decisions compared to written advance directives. This article examines the role video advance directives play in advance decision-making and their legal and practical implications to the existing framework. An appreciation of the legal challenges presented by this development facilitates an understanding of their use in contemporary advance directives and enables appropriate recommendations for implementing safeguards in their use.
\end{abstract}

Keywords Advance directives · Capacity $\cdot$ Recording technology $\cdot$ Safeguards ·

Video

\section{Introduction}

Creating and implementing care preferences are increasingly significant issues in providing healthcare services that are consistent with patient-centred care. ${ }^{1}$ A longer life expectancy and greying population, with rising numbers of people

\footnotetext{
1 Silveira et al. (2010: 1211), Umar et al. (2012: 1), Brom et al. (2014: e100435), Say and Thomson (2003: 542), Sepucha and Mulley (2009: 53S).

Hui Yun Chan

h.chan@hud.ac.uk

1 Department of Law, Huddersfield Business School, University of Huddersfield,

Queensgate, Huddersfield HD1 3DH, UK
} 
experiencing incapacity at various stages of their lifespan presented important challenges to healthcare services. Medical advancements in life-sustaining treatments and the growing recognition of patient autonomy provide the impetus for stronger patient voice through advance directives (ADs). ADs offer the opportunity for people to express their consents or refusals regarding treatment decisions intended to be legally binding on healthcare professionals when they become incapacitated at a future point of time. In England and Wales, an advance directive refusing treatment is known as an advance decision. ${ }^{2}$

The moral arguments for and against ADs have figured primarily in response to the possibilities of medical advancements in prolonging life. ${ }^{3}$ The AD debate is generally prevalent in the Anglo sphere and European countries where the literature explored the legal and ethical issues surrounding end-of-life decision-making. ${ }^{4}$ There is a scarcity of comprehensive evidence regarding the use of ADs, however, a country report on ADs compiled in 2008 on the regulatory state of ADs comprising European countries and the USA revealed varying patterns of awareness, use and legislative approaches towards ADs. ${ }^{5}$ For example, the Report revealed a common theme on the extent to which ADs are being used in practice; with a higher level of awareness and use of ADs reported in Germany, the Netherlands and the USA compared to other European countries such as Spain, France, Italy, Switzerland and UK. ${ }^{6}$ Australia and New Zealand have also engaged in promoting the use of ADs and advance care planning in the population. ${ }^{7}$ An European-wide report published a decade later highlighted the continued need for member states to advance efforts towards supporting self-determination through powers of attorney and ADs, in which the latter is noted as underdeveloped. ${ }^{8}$ These developments demonstrate that while the populations appreciate the benefits of making ADs for end-of-life decision-making which are supported by efforts to boost their use, the uptake has not increased significantly. This may be potentially attributed to the difficulty in articulating the wishes in writing or other existing considerations in accessing these services.

Currently, there is no uniformed format for ADs and each written or printed form would either depend upon the format available from hospitals, or care providers or in downloadable format from websites operated by charities. ${ }^{9}$ Oral ADs, on the other

\footnotetext{
2 S24 Mental Capacity Act 2005 (UK).

3 See for example Maclean (2006: 291), Maclean (2008: 1), Mitchell (2012: 245), Ryan (1996: 95), Buford (2008: 423), Dresser (1995: 32).

4 Veshi and Neitzke (2015: 321), Olick (2012: 232), Horn (2014: 59), Silveira et al. Piette (2014: 706).

5 Brauer et al. (2008).

${ }^{6}$ Brauer et al. (2008). Recent publication also supported this finding where ADs developed differently according to different regulatory approaches and binding force of ADs in the European regions: Simón (2011: 75).

7 Ruseckaite et al. (2017: e018024), Duckworth and Thompson (2017).

${ }^{8}$ Ward (2018: 5).

9 An example is the Compassion in Dying organisation that provides guidance for the completion of online forms in England and Wales.
} 
hand, are wishes expressed to another without being reduced to writing. ${ }^{10}$ Although there is no requirement that an $\mathrm{AD}$ has to be in writing under the common law prior to the Mental Capacity Act $2005,{ }^{11}$ the good practice is to have oral expressions of wishes reduced to writing as evidence of its existence. ${ }^{12}$ As such, the written format is the preferred mode for the purposes of proving the existence of such wishes.

A recent development revealed the emergence of video ADs as an option that aims to strengthen a person's expression of wishes in the light of the challenges arising from written and oral ADs. ${ }^{13}$ This development gives rise to important questions in the advance decision-making framework: What are their roles in the ADs landscape? Do they offer a better alternative to the current practice of written ADs? What are the challenges, and legal and practical implications arising from a video recording expressing an $\mathrm{AD}$ for treatment preferences? These questions illustrate the type of new and important concerns with alternative modes of expressing treatment wishes and are the focus of this paper. A consideration of these legal issues is appropriate and timely, in tandem with the evolving ways in creating ADs. The paper aims to examine the consequences of emerging forms to create ADs such as video recordings compared to the conventional written form. It provides an addition to the knowledge on ADs, their binding or non-binding character and the related questions of ensuring their validity or ways of applying them in problematic situations where the patients are unable to state their preferences.

In answering these questions, "Recording Care Preferences Through Videos: A Turning Point for Advance Directives?" section examines the use of video ADs and sets out the key differences between existing forms of ADs and video ADs. It then assesses the extent to which visual representation of ADs can affect a significant shift in the ADs landscape. "An Evaluation of Video ADs: Roles, Implications and Safeguards" section evaluates the role of video ADs, particularly what role it should play and analyses the legal and practical challenges associated with recording, storing, accessing and authenticating video ADs. Their implications are discussed, followed by recommendations to address the challenges of this new medium. While this option appears to offer an attractive alternative to communicate their wishes in a more compelling manner than written ADS, the discussion reveals that video ADs

\footnotetext{
${ }^{10}$ W Healthcare NHS Trust $v$ H and others [2004] EWCA Civ 1324. This decision demonstrated the difficulties with establishing the validity and applicability of an oral AD pertaining to refusing life-sustaining treatment.

11 The Mental Capacity Act 2005 now requires advance decisions refusing life-sustaining treatment to be written, signed, witnessed and verified to the effect that they would apply even at the risk of death: s25(5).

12 Paragraphs 9.22 and 9.23 of the Mental Capacity Code of Practice has clarified the position of oral ADs to the effect that healthcare professionals should record oral ADs in the person's medical record which would prevent future confusion regarding decisions made. Such records should clearly state when the decision would become applicable, the treatment to be refused and additional details of people who were present when the oral ADs were recorded.

13 Examples of websites offering electronic ADs and video ADs: In My Own Words inmyownwords. com. Accessed 5 February 2019; My Directives https://mydirectives.com/. Accessed 5 February 2019; and Compassion in Dying https://compassionindying.org.uk/. Accessed 5 February 2019 which provides the option to create personalised ADs.
} 
suffer from various similar challenges faced by written ADs. Although video ADs are currently offered in the USA, its historical development suggests that it would not be too improbable to consider its expansion to other parts of the Anglo sphere and European jurisdictions, as such; there are important broader implications for countries considering this form of video ADs. Reference is made to the law in England and Wales to provide a contextual background to the following discussion and analysis.

\section{Recording Care Preferences Through Videos: A Turning Point for advance directives?}

Video ADs are designed to communicate a person's wishes through visual, moving images containing the person's facial expression, gestures and voice. It is a relatively new development because of increasing availability of various online platforms offering services for creating ADs. ${ }^{14}$ This development demonstrated the evolution of ADs from existing paper-based documents (written or printed) to videos, electronic health records and online registries, in response to technological advancements. ${ }^{15}$ The close nexus between increasingly embedded technology use in daily life, and the technology literacy rate of older people merit closer consideration in the context of newer forms of expression of wishes. The Eurostat figures revealed that in 2016 , about $45 \%$ of the elderly population of 65 years and above used the internet at least once a week although a minority used social networks on the internet. ${ }^{16}$ A disparity exists between different European states, for example, a higher proportion of the elderly population in the northern and western parts of Europe used the internet compared to their southern and eastern counterparts. ${ }^{17}$ Similarly, in the UK, studies have shown that an increasing number of older people have started engaging with technology, with growing use of online applications either on the computer or on their smart phones. ${ }^{18}$ The number of older adults over 65 years old using smart phones and tablets and having social media profiles have also increased. ${ }^{19}$ Despite rising numbers of technologically savvy older adults, there remains a proportionate number that are not confident in navigating the online world, particularly in managing personal data, lacking in knowledge on privacy and confidentiality issues. ${ }^{20}$ It

\footnotetext{
14 Examples of services offering online forms for ADs include: Advance Decisions Assistance http:// adassistance.org.uk/write-it/. Accessed 12 March 2019; My Decisions https://mydecisions.org.uk/. Accessed 12 March 2019. In the case of My Decisions the final documents need to be printed off, signed and shared with family members, relatives, doctors or those that are close to the person. The Maryland state in the USA allows ADs to be created in the form of video recording. See Marso (2017).

15 Klugman and Usatine (2012: 657).

16 Eurostat (2019).

17 Eurostat (2019).

18 Ofcom (2017, 2018); Independent Age, 'Older people, technology and community: the potential of technology to help older people renew or develop social contacts and to actively engage in their communities' https://www.cisco.com/c/dam/en_us/about/ac79/docs/wp/ps/Report.pdf. Accessed 2 January 2019.

19 Ofcom (2018).

20 Ofcom (2018).
} 
is also possible to postulate at this point the link between higher prevalence of ADs and internet use in the northern and western parts of Europe compared to southern and eastern parts of Europe. These trends potentially influence their choices in making video ADs as their understanding and expectations of these issues would bear upon their decisions to express their wishes through video recordings.

Empirical studies and the literature thus far have appeared to demonstrate positive outcomes for video messages as potential supporting tools in decision-making. ${ }^{21}$ Limited studies revealed that videos portraying treatment discussions shown to people prior to them becoming patients could facilitate their understanding of expressing patient wishes for end-of-life interventions. ${ }^{22}$ This approach helps inform the patients about certain health conditions and illnesses in order to foster knowledge and understanding by depicting various health circumstances (e.g. vegetative states; coma) or different goals of care and palliative care options. ${ }^{23}$ Any negative perceptions towards the outcomes of these conditions could be remedied with doctors' consultations or the provision of further information. ${ }^{24} \mathrm{~A}$ separate study highlighted that adding a video message helped clarify patient wishes and enabled doctors to achieve consensus on resuscitation decisions compared to the existing living wills and do-not-resuscitate code. ${ }^{25}$ Patients who utilise videos as support tools in decision-making are more likely to choose comfort care and retain this preference compared to patients who do not utilise video decision aids. ${ }^{26}$ Advocates of video ADs also argued that patients are better able to appreciate various health conditions as the circumstances progress, utilising the strength of communicating information visually compared to narratives. ${ }^{27}$ It can be postulated that the results in the available studies conducted might be true for patients with dementia but may not be extended to other patients owing to the distinctive nature of progressive decline in dementia, compared to other terminal illnesses.

While studies have investigated the application of video decision aids to facilitate people expressing their wishes in anticipation of dementia, ${ }^{28}$ little has been explored regarding video ADs as instruments in expressing treatment preferences in the same standing as written ADs and their legal and practical implications. This aspect warrants closer consideration to identify its potential impact in the light of developments made in improving ways of communicating a person's wishes. Advocates of video ADs argued that audio-visual recordings could communicate better preference decisions compared to written ADs, due to the inadequacies of written ADs in communicating the meaning of the decision and emotions of the person, as they

\footnotetext{
21 Volandes et al. (2009: b2159), Jain et al. (2015: e007491), Deep et al. (2010: 229), Moseley et al. (2005: 211), Sudore et al. (2014: 674), Toraya (2014: 942), Vearrier (2016: 339).

22 Mirarchi et al. (2017: 51), Sandza (2017).

23 Deep et al. (2010: 229), Wilson et al. (2015: 621).

24 Volhard et al. (2018: e0197229).

25 Mirarchi et al. (2017: 51).

26 Volandes et al. (2009: b2159).

27 Deep et al. (2010: 229).

28 Hanson et al. (2017: 24), Volandes et al. (2009: b2159), Mitchell et al. (2018: 961), Volhard et al. (2018: e0197229).
} 
are often presented in legalistic languages. ${ }^{29} \mathrm{~A}$ further argument in favour of video ADs is its potential in enabling family members and doctors who encountered the patients' expression of wishes for the first time to identify the patients' wishes for future care, including their explanation of the choices on limits of care or substitute decision-makers. ${ }^{30}$

Additionally, supporters of video ADs viewed their potential in radically improving the way end-of-life decisions are recorded, citing the increasing use of technological advancements in healthcare services. ${ }^{31}$ A video AD illuminates family understanding about the patient's decision thereby increasing acceptance of the patient's wishes, and obviating the need for doctors to reconfirm such wishes with family members. ${ }^{32}$ This claim is doubtful because ADs are not static, and the time lag between the making of and implementing the $\mathrm{AD}$ would mean that changes would have occurred necessitating reviews and verification of the surrounding circumstances. Another argument is that video ADs provide the opportunity to verify the act of making the $\mathrm{AD}$, while simultaneously enabling doctors to validate the person's mental capacity in expressing their wishes or refusals. ${ }^{33}$ Some anecdotal evidence, from both patients and their relatives that video ADs have provided clarity to their wishes supports this argument. ${ }^{34}$

A video $\mathrm{AD}$ is said to resemble a contemporaneous doctor-patient discussion in care planning, which demonstrates the strength of a patient's wishes and offers a better understanding of the decision-making process by virtue of their reasoning and choices. ${ }^{35}$ This claim is arguable, as there are still subtle differences between a real time conversation and a one-way communication done through a different medium. The strength of the expressions of wishes and the effect upon which such expressions have on the listeners and viewers varies; however, it does not automatically mean that because there is a change in the medium in communicating these wishes that it demonstrates the strength of the patient's wishes. It is important to recognise that not all patient wishes are binding only because they are found in ADs, regardless of the medium. Therefore, despite this perceived advantage and some positive outcomes for advance care planning, these benefits have not been empirically confirmed thus far. Further research is essential in confirming the claims advanced by the advocates of video ADs and any anecdotal evidences.

In order to establish the extent to which video ADs is better in providing clarity to patient wishes and to appreciate their legal and practical implications, it is essential to consider the differences between existing forms of ADs and video ADs. These differences are considered from the perspectives of forms and format, storage,

\footnotetext{
29 Moseley et al. (2005: 211, 213).

30 Ibid.

31 Moseley et al. (2005: 211, 216).

32 Ibid.

33 Ibid.

34 Testimonials which appeared on the website of the video AD recording provider, inmyownwords.com: http://inmyownwords.com/testimonials.html. Accessed 12 March 2019.

35 Ibid.
} 
access and current regulatory status. The prevalent forms of ADs will be considered first, followed by video ADs.

\section{An Analysis of Key Differences Between Prevalent Forms of ADs and Video ADs}

\section{Prevalent Forms of ADs}

\section{- Written/printed forms}

A majority of ADs are in written or printed forms and especially crucial in ADs refusing life-sustaining treatments. ${ }^{36}$ Although there are no specific requirements for ADs to be in paper format or physical copies, the formal requirements attached to specific ADs meant that people are encouraged to reduce their wishes into writing. ${ }^{37}$ A written AD not only evidences the person's intention regarding the existence of such wishes to healthcare providers, but is particularly acute in instances where it has to be verified to the effect that it would apply even if life is at risk. Cases in England and Wales have demonstrated the importance of written ADs when disputes arose regarding treatment decisions. ${ }^{38}$ ADs can range from values statement containing short paragraphs or combined with tick boxes for refusals of specific medications or medical interventions. Some advance care plans are more elaborate and available in workbook format with guided instructions for completion, and for charting the progress as patients reflect upon and record their wishes.

The structure and format of these forms would depend upon the template available to the respective hospitals or care homes, adapted to and guided by the policies established by the organisations or following the requirements of the prevailing law. ${ }^{39}$ For example, Compassion in Dying, a charity registered in England and Wales provides the opportunity for people to create ADs which can be completed online, printed out and signed while Macmillan Cancer Support also has its own

\footnotetext{
36 Special requirements for ADs refusing life sustaining treatment were introduced to "provide[s] a safeguard that the life of the patient will always be preserved in matters of doubt": Lord Chancellor's Department (1997: [4.26], [4.27]).

37 See for example as indicated in the Mental Capacity Code of Practice regarding the necessity of creating written records of ADs expressed orally.

38 Sections 25(5) and (6) Mental Capacity Act 2005 (UK); Re AK (Adult Patient)(Medical Treatment: Consent) [2001] 1 FLR 129 where AK's AD was communicated to his doctors through blinking his eyes and recorded in writing; in NHS Trust v T (adult patient: refusal of medical treatment) [2004] EWHC 1279 (Fam), blood transfusion can be considered as life-sustaining treatment and would need to be in written form; in $\operatorname{Re~D}$ [2012] EWHC 885 (COP) the letter which was written and signed by Mr D expressing his wishes to refuse life-sustaining treatment was given weight in considering treatment withdrawal.

39 Examples include: Advance Care Plan http://advancecareplan.org.uk/examples-document-templates/ and http://advancecareplan.org.uk/advance-care-plan-passport/. Accessed 5 January 2019. The site also allows people to have to option to create passport-sized advance care plan.
} 
customised AD forms which are adapted from the National End-of-Life Care Programme. ${ }^{40}$ Specific forms are also in use across the different States and Territories in Australia and the USA, which adhered to the particular legal requirements under the respective laws in the jurisdictions. ${ }^{41}$ The Australian AD forms are similar to the English ones to the extent that they need to be completed, printed and signed by the person.

\section{- Storage: kept at solicitors' offices or in undisclosed places}

Once ADs are made, they may be kept at various places, ranging from the solicitor's office, in wallets or purses, on the fridge, worn as emergency bracelets or necklaces linked to ambulance services. In some extreme cases, instructions are tattooed on the visible parts of the body such as across the chest or arms. Oral ADs may not be known until they are communicated to the doctors or the courts by family members, relatives or friends when questions about the continuity of treatment arose, by which time the patient is already incapacitated. ${ }^{42}$

\section{- Access: may/not be found}

Access to ADs is a major challenge in clinical practice often linked to the ways they are stored or the lack of awareness regarding their existence. ${ }^{43}$ They may not be found or, if found, discovered too late while decisions were made and treatments continued to be administered. The recent decision of Brenda Grant in England

\footnotetext{
${ }^{40}$ Compassion in Dying AD online forms https://compassionindying.org.uk/choose-a-way-to-make-anadvance-decision-living-will/, https://compassionindying.org.uk/library/advance-decision-pack/ and https ://mydecisions.org.uk/?utm_source=cidwebsite\&utm_medium=planningahead\&utm_campaign=CIDPL ANAHEAD. Accessed 5 January 2019. See also Macmillan Cancer Support Advance Decision to Refuse Treatment: https://www.macmillan.org.uk/_images/ADRTDocument_20170505_JS_tcm9-311758.pdf. Accessed 5 January 2019.There are also other forms which are used by different NHS in England and Wales, see for example: http://www.npmc.nhs.uk/website/K82016/files/Living\%20will\%20Forms\%20for \%20patients\%20update\%202018.pdf. Accessed 14 March 2019.

${ }^{41}$ In Australia, different AD forms are in use. The Advance Care Planning Australia organisation provides information on the different requirements of the law for completing ADs: https://www.advancecar eplanning.org.au/individuals/advance-care-directives-and-the-law. Accessed 10 March 2019. Separate websites provide individual AD forms for download and printing, for example, the South Australia AD form: https://advancecaredirectives.sa.gov.au/forms-and-guides. Accessed 10 March 2019; Western Australia AD forms: http://www.health.wa.gov.au/docreg/education/population/HP11536_advance_health_ directive_form.pdf. Accessed 10 March 2019; New South Wales AD forms: https://www.health.nsw.gov. au/patients/acp/Publications/acd-form-info-book.pdf. Accessed 10 March 2019; Victoria AD forms: https ://www2.health.vic.gov.au/hospitals-and-health-services/patient-care/end-of-life-care/advance-care-plann ing/acp-forms. Accessed 14 March 2019). In the United States, an interest group, AARP has put together various ADs forms according to the different states: https://www.aarp.org/caregiving/financial-legal/freeprintable-advance-directives/ accessed 14March 2019.

${ }^{42}$ An example is $W$ Healthcare NHS Trust $v H$ and others [2004] EWCA Civ 1324 where questions arose regarding the validity of the alleged orally expressed wishes and the context in which they were made.

${ }^{43}$ England and Wales reported lack of knowledge regarding ADs among the population and by medical professionals, and measures are implemented to ensure any ADs are retained with patients' medical records: Ward (2018: 44).
} 
illustrates this point. The hospital was fined for $£ 45,000$ for contravening the AD due to their inability to access the AD refusing treatment in a timely way to implement her wishes, resulting in her receiving life-prolonging treatment for more than a year which was against her clearly stated wishes. ${ }^{44}$ It transpired that the AD was hidden beneath copies of old medical records which could not have been easily discovered. Additionally, Brenda's children and nursing home were neither informed about nor in possession of copies of her AD. The hospital has since apologised to her family and implemented new filing systems to remedy the inadequacies.

\section{- Regulated under specific laws}

ADs are governed under specific legislations in some jurisdictions. The Mental Capacity Act 2005 applicable in England and Wales gives advance decisions refusing treatment a legally binding status while Australia, Canada and Singapore have also granted specific ADs legally binding status in their respective statutes. ${ }^{45}$ The laws outlined formal requirements for ADs to be valid and applicable to the circumstances. ${ }^{46}$ Failure to comply with these laws would result in the wishes not having a legally binding effect but would nonetheless be taken into account in treatment decisions. $^{47}$

\section{Video ADs}

\section{- Video/audio format}

Video ADs contain images and voice recordings depicting people expressing their wishes. There is currently no uniformed mode of recording, resulting in a range of length, quality and content of recording, and questions about who would be involved in the conversation. Consequentially, a video AD could also record patients in conversations with their doctors, families or friends engaged in the process of making the AD.

\footnotetext{
${ }^{44}$ Paduano, 'Payout after woman was kept alive against her will' (BBC, 6 December 2017) https://www. bbc.co.uk/news/uk-england-coventry-warwickshire-42240148 accessed 14 March 2019.

${ }^{45}$ Sections 24-26 Mental Capacity Act 2005 (UK); Medical Treatment Planning and Decisions Act 2016 (Victoria, Australia); Powers of Attorney Act 1998 (Qld); Advance Care Directives Act 2013 (SA), Advance Personal Planning Act 2013 (NT), Medical Treatment (Health Directions) Act 2006 (ACT); Guardianship and Administration Act 1990 (WA); Health Care (Consent) and Care Facility (Admission) Act RSBC 1996 c 181, Personal Directives Act 2000 RSA c P-6, Advanced Health Care Directives Act 1995SNL c A-4.1, Personal Directives Act 2005 SNWT c 16, Personal Directives Act 2008SNSc 8, Consent to Treatment and Health Care Directives Act 1988 PEI c C-17.2, An Act respecting end-of-life care Bill 2014 (52) c 2 (Quebec), the Health Care Directives and Substitute Health Care Decision Makers Act 1997 S c H-0.001, the Health Care Directives Act 1992 CCSM c H27; Health Care Consent Act 1996 SO c2; Advance Medical Directives Act 1996 (Sg).

${ }^{46}$ For example, s 25(5) of the Mental Capacity Act 2005 stipulates specific requirements for a binding advance decision refusing life-sustaining treatment.

${ }^{47}$ For instance, in Re D [2012] EWHC 885 (COP), although the signed letter refusing life-sustaining treatment was not verified to the effect that it is to apply even if life is at risk, the court found that it was persuasive when considering treatment withdrawal from $\mathrm{D}$.
} 
- Storage: stored either personally or on online platforms hosted by third parties

Video ADs can be stored on a wide range of devices, for example, on mobiles, tablets, laptops, desktops, cameras or on the cloud as well as on portable drives carried on the person. Online versions may be downloaded for offline viewing or printed off for further actions (e.g.: discussion, review, and updating), or shared between specific people such as family members, relatives or friends. Websites hosted by third parties offering services for personalised video ADs may also offer storage facilities. Recordings that are created, uploaded and stored on these online platforms will give rise to questions of access, safety, authenticity and currency of the ADs. While some of these concerns may be obviated with password encrypted measures, provided the person has notified relevant people that such recordings exist, other questions remain. This aspect will be dealt with in more detail in "An Evaluation of Video ADs: Roles, Implications and Safeguards" section.

\section{- Access: where and by whom}

A related concern with storage is the accessibility of these video ADs. An advantage of the portability of video ADs is the likely ease of accessibility by family members, relatives, substitute decision-makers, doctors or solicitors. On the other hand, if they were not informed of the whereabouts of such recordings they may not be as accessible as initially anticipated. It would become complicated where these recordings are password protected and the person has now become incapacitated to provide any further information. Video ADs that are stored on third party websites would likely be accessible by doctors or hospitals that are registered with the services and upon notification by either the patient or relatives of the existence of such recordings.

The key challenge here is accessibility in a timely manner. Knowledge regarding the existence of such ADs is a prerequisite to accessing the videos which would allow them to be implemented. Additionally, in the event that the video files became corrupted and could not be opened for viewing, their wishes would be rendered futile. Questions concerning confidentiality and system security equally raise important implications; for example, the likelihood of video ADs being tampered with, or liable to manipulation. Crucially, the infrastructure that supports the timely access of these video ADs warrants closer investigation.

\section{- Regulated?}

As a relatively new development, video ADs are currently not specifically addressed within the scope of the law. Similarly, there are no decided cases on video ADs. However, it is likely that if video ADs could meet the requirements of validity and applicability of ADs under existing rules, their use would not be automatically excluded. 


\section{The Extent to Which Video AD is a Turning Point in the ADs Landscape: A Better Tool in Expressing a Person's Wishes?}

Some preliminary observations can be offered arising from a consideration of the key points above. Video ADs differ from written ADs in the medium they are communicated and the effect they have on the viewers. In this sense, it provides an alternative to people who would prefer to convey their preferences governing future care and treatment using visual images. The advocates of video ADs highlighted the strength of video ADs in communicating better preference decisions compared to written ADs, particularly where they enable people to explain their choices regarding the decision and to nominate substitute decision-makers. This claim is not exclusive to video ADs and thus not a particularly "better" medium, because a written AD could also incorporate these elements. Therefore, a well-written AD that is both valid and applicable is adequate in articulating the person's wishes. Similarly, a well-scripted and clearly narrated video AD can be accepted as valid and applicable. Additionally, the law generally does not prescribe the mode in which people express their wishes; but rather the decision itself, which encompasses their capacity, voluntariness and an appreciation of the nature and consequences of the decision. ${ }^{48}$ While it is not a requirement that people should explain their choices, decisions that are perceived as irrational would be subject to questions. ${ }^{49}$ It is equally important to highlight the writing process involved in creating ADs. The process of writing ADs, while difficult, enables people to clarify their thoughts over time, compared to videos where they need to be clear about what to record at that time. ${ }^{50}$ This would be problematic for people who do not know what they would want to refuse and would prefer to have their preferences reduced to writing.

Video ADs are said to communicate stronger convictions regarding end-oflife decisions on the basis that doctors are able to confirm the person's capacity and intentions compared to written ADs where doctors would require further evidence from other treating doctors, nurses or family members. ${ }^{51}$ This would presuppose a recording made during doctor-patient consultations, which would provide the opportunity for such assessments to take place, or where the doctors undertake to review the video recording later. In case of the former, it would provide clear evidence at the time the $\mathrm{AD}$ was made that the patient had capacity,

\footnotetext{
48 See for example s 25(1) of the Mental Capacity Act 2005.

49 For instance NHS Trust v T (adult patient: refusal of medical treatment) [2004] EWHC 1279 (Fam) illustrated the difficulty with accepting a patient's decision who perceived her blood as evil and had sought to refuse further blood transfusion by making specific AD refusing blood transfusion. In $R e C$ (refusal of medical treatment) [1994] 1 WLR 290 (Fam), a psychiatric patient was judged as having capacity to refuse amputation of his leg at the risk of death despite the availability of surgical procedures to save his life.

${ }^{50}$ In $\operatorname{Re} D$ [2012] EWHC 885 (COP), the written letter signed by the patient demonstrated the thought process involved in anticipating the type of life and treatments which the patient wished to avoid. Similarly, in Re AK (Adult Patient)(Medical Treatment: Consent) [2001] 1 FLR 129 illustrated the painstaking procedures involved in recording the treatment refusal which ensured that it corresponded with AK's wishes, having considered the medical prognoses.

51 Moseley et al. (2005: 211).
} 
as assessed by the doctor present in the recording. In terms of the latter, unless the patient's surrounding circumstances have unduly influenced the capacity to decide, the presumption of capacity ought to apply to video ADs. Further, uncertainties may also arise surrounding the circumstances in which the video recordings were made, particularly where the patient recorded such videos privately and not revealed until they were sought to be implemented. This situation would be very similar to a written AD but communicated through a different medium.

The effect conveyed by visual images and the voice of the patient may add to the person's conviction in refusing or consenting to particular treatments, however, would such visual representations deliver similarly clear message as written ADs? This would depend upon whether the wishes are clearly set out and what would be important for doctors to know in implementing the AD. A written AD, when presented, can be swiftly scanned and read for salient points in determining whether to withdraw or withhold treatment according to the patient's wishes. A video $\mathrm{AD}$ may not benefit from this treatment because the entire video needs to be viewed (and reviewed for confirmation) and notes taken to ensure salient points are extracted from the video, which cumulatively takes longer to interpret. Nevertheless, as suggested above, a clearly narrated video AD would provide clarity in terms of what the patient would have wanted in the circumstances.

Written ADs and video ADs suffer from questions of accessibility when called into implementation. Their existence would only become known if the patients have informed the doctors, family members or relatives, as illustrated from the comparison above. However, an advantage of the video AD is the availability of service providers that would be able to permit access to doctors and hospitals registered to these services where these videos are linked to the patient's medical record. It is appealing from this perspective for those who prefer the convenience afforded by the connectivity between the healthcare providers and the video recording service providers. It is envisioned that prior to the recording there would be substantial preparatory work in scripting the wishes, clarifying what the person would have wanted, recording and uploading the videos upon completion. The process of writing ADs on the other hand would seem comparatively less onerous than creating video ADs. The Brenda Grant example above demonstrates that written ADs can only play a role when they can be accessed in a timely way and that people are informed about it. Video ADs may also fall through the gap if their existence is unknown. However, where they are recorded and stored in the hospital system with a note (physical copy or virtual) appended to the medical records that such videos exist, this measure removes an obstacle in access. The case also signifies the importance of informing people once the AD is created, as evidenced by the following:

My personal experience with my mother and my aunt, (both of whom had advanced dementia at the time), is that without me physically taking a copy of their $A D s$ to the hospital every time they were admitted, and ensuring that the doctors were aware of them, the documents would have been ignored. Once doctors saw the AD, I generally found them very helpful in restricting treatment. In my aunt's case, the consultant stopped all life-preserving med- 
ication immediately, in accordance with her wishes expressed in her AD.

Without my intervention this wouldn't have happened. ${ }^{52}$

The sentiment above also highlighted another potential challenge for video ADs. Where they are filed in the online system but inadvertently obscured by other files, they are easily overlooked unless doctors are alerted to their existence prior to implementation. Storage of video ADs, as such, is an important consideration closely connected to the question of access. Questions would inevitably surface regarding the security and reliability of these recordings where they are being stored on these platforms. Patients would expect that their ADs are not only securely stored but also accessible by healthcare providers when needed. Consequently, questions arise as to how well prepared are healthcare providers in implementing the system and how integrated are these healthcare services regarding accessing these ADs in a timely manner. Without appropriate infrastructures and integrated facilities, the confidence in using video ADs may be low. That said the same challenge would also confront written ADs. If video ADs were to be accepted, there should be progress towards electronic health records, or an equivalent developed system to implement such provision of services.

There are also broader questions regarding the effective articulations of care preferences in video ADs. This entails questions concerning how the recording is made, the quality of such recordings, the prevailing technology literacy rate, the extent of inequality in using these modes of recording, and the burdens of typing, uploading and transcribing them. These concerns are important especially where they are intended to be offered as a choice in communicating treatment preferences for future incapacity and promoted to the population. There are both advantages and drawbacks with video ADs compared to written ADs. While its reach may yet to be accurately predicted, where there are significant progress in migrating health records from paper-based systems to online systems, people would be more confident in using video ADs. It is clear that video ADs have offered an option that could improve the way ADs are communicated and evidencing a person's treatment preferences at the end-of-life, however they are subject to various external factors that would influence its use and application, as demonstrated above. It is yet to be seen that video ADs have shifted significant grounds in the ADs landscape, although its use cannot be discounted.

The European Council Report recommended further research and education of ADs at a European level, indicating the possible extension of video ADs as an option to strengthen the people's instructions for future care. ${ }^{53}$ It is anticipated that any increase in using video ADs would be gradual rather than exponential. Sound architectural support in the healthcare system that makes access and communication

\footnotetext{
52 Phil Cheatle in recounting the reality of ADs implementation: MDMD, 'Hospital trust pays out $£ 45,000$ for ignoring an Advance Decision’ (My Death My Decision, 6 December 2017) https://www. mydeathmydecision.org.uk/hospital-trust-pays-45000-ignoring-advance-decision/. Accessed 14 March 2019.

53 Ward (2018: 6, 7).
} 
seamless and a normalisation culture in using video ADs would be promising conditions to allow the benefits of video ADs to emerge.

\section{An Evaluation of Video ADs: Roles, Implications and Safeguards}

In this section, I assess the extent to which video ADs should have a role in advance decision-making and if so what function it could perform. Having identified that video ADs can play a role in communicating treatment preferences, I evaluate the legal, practical and clinical implications arising from such practices. Based on this evaluation, I propose several safeguards to address the concerns raised in the use of video ADs.

\section{A Consideration of the Role of Video ADs}

Video ADs provide an option to communicate a person's wishes and while its use is still in infancy, it should have a role to play with additional safeguards. Video ADs offer insights into the person's wishes through demonstrating their convictions to a particular decision, which are comparable to conveying the wishes to family members, friends or doctors in informal or formal settings. If such wishes were subject to judicial disputes, the video recording could be played out as evidence, either supporting or disclaiming any disputed issues. Thus, it would be subject to scrutiny, comparable to written ADs, where the video recording could be paused or re-played for clarification of points. The content of the video ADs could be transcribed for further confirmation or explanation, which would be helpful in decisionmaking. This mode will appeal to either technology-conversant users or users from a younger demography thinking of engaging with future medical treatment and care preferences.

Should video ADs replace the existing form of written or printed ADs in its entirety, or should it perform a supplementary role in enhancing the person's communication of wishes? First, we need to consider the implications attached to the different roles video ADs play in relation to the question above. Where video ADs are intended to supplement written ADs, they would be comparable to values statement, where additional information or explanation that are precluded from written ADs could be incorporated and further expanded. The video recording can also operate as a summary to the written AD capturing the person's wishes or highlighting any other pertinent information. Questions surrounding the recording process and the involvement of relevant people ought to be clarified in order to pre-empt any external circumstances from potentially compromising the strength of this evidence.

Conversely, if video ADs were to be treated as replacing written ADs, detailed consideration should be given as to how they should be recorded, what should be included and who should be involved in the creating process. For example, should it always be recorded in the presence of others (such as trained advance care planning nurses, health and social workers, families or support person), and doctors, psychiatrists, specialists or other allied healthcare professionals? Should people record 
video ADs individually or with doctors or a support person? These questions are significant because an advantage in recording the video AD in the presence of doctors or allied healthcare professionals is that there is a greater chance that important and relevant information are included in the recording. This is achieved either through the patient receiving material information about diagnosis and prognosis or having the opportunity to appreciate the nature and consequences of each proposed treatment option. Another benefit is the opportunity to ascertain and verify the level of mental capacity of the person concerned should any doubts arise. This setting would entail a more formal and structured system to record the wishes compared to a recording made individually and outside of clinical settings. People who prefer the privacy of recording the wishes at home would then need to have their video ADs reviewed by their doctors, or qualified professionals. If they were recorded in the presence of carers, support person, family members or relatives, care should be taken of any presence of undue influence on the person, similar to making written ADs. ${ }^{54}$ If it was recorded individually, it is paramount to consider whether the person is informed, and the validity is established. ${ }^{55}$ The growth of specialist ACP facilitators or nurses trained in conducting ACP is a resource that can be tapped into to offer this type of services, which would be realistically applicable only in the foreseeable future given the level of development in healthcare services. While video $\mathrm{ADs}$ may be perceived as viable alternatives to written ADs, they are not the panacea for the challenges involved in advance decision-making generally or written ADs specifically. Video ADs as supplementary to written ADs would also be subject to similar considerations above, albeit on a less extensive scale. It is thus essential to clarify the relationship between video ADs and the prevailing practices and legal framework, including the relative importance of video ADs in specific contexts and the role they play.

Given the preceding assessments, it is clear that a video AD performs a viable supplementary role that renders additional support to a person's written AD. A video $\mathrm{AD}$, is, to an extent, akin to oral $\mathrm{ADs}$ but with the benefit of a captured visual image depicting the person's demeanour at the time with accompanying words expressed to the intended viewers and capable of being replayed verbatim compared to recollecting conversations communicated orally which would be subject to doubts through the passage of time. ${ }^{56}$ Videos that record conversations between the patient

\footnotetext{
${ }^{54}$ In $R e T$ [1992] EWCA Civ 18, there were questions surrounding the voluntariness of the patient who had expressed refusal to blood transfusion under the influence of her mother. The patient's genuine will was thought to have been overpowered in her weakened physical and mental conditions.

55 In W Healthcare NHS Trust $v H$ and others [2004] EWCA Civ 1324, the court found that there were no medically qualified witnesses who had discussed with the patient the implications of slowly starving to death over a 2-to-3-week period if she were to decide on the course of refusing the feeding tube. Similarly in $\operatorname{Re} T$ [1992] EWCA Civ 18, it was found that the patient was misinformed about other available options when she refused blood transfusion and a failure to appreciate the risks, nature and consequences of refusing blood transfusion.

56 In W Healthcare NHS Trust $v H$ and others [2004] EWCA Civ 1324, the court found that the conversations regarding the patient's quality of life generally had occurred many years ago before she was admitted to the care home and none of them dealt specifically with the withdrawal of feeding. This formed one of the grounds upon which the court found any prior expressed wishes to have lacked the clarity and specificity of an AD refusing life-sustaining treatment.
} 
and the doctor or with other allied healthcare professionals in the process of creating the $\mathrm{AD}$ can also be used to clarify or challenge any misconceptions or ambiguity in interpreting the patient's written AD. As such, it becomes a valuable source should disputes occur regarding the person's state of mind at the time of communicating the $\mathrm{AD}$. Additionally, written ADs and physical records of medical notes remain the prevailing norms of evidence in making treatment decisions.

Video ADs should only become mainstreamed where the majority of people can access them and the healthcare infrastructures are prepared to support their implementation. This includes considerations such as the time needed to interpret the video and doctors' knowledge in implementing them. Clinical realities may prevent their timely accessibility and interpretation. Guidelines on video ADs would be helpful to offer clarity on their use, particularly where the format is still subject to individual provider's determinations and the person's favoured choice and settings. As a supplement, video ADs can strengthen, clarify or offer additional explanation and information to the written ADs made by the patients. As standalone evidence, it may not necessarily perform better than written ADs in expressing treatment decisions. The advocates of video ADs seemed to support their supplementary role to written ADs in order to address the limitations of the latter. ${ }^{57}$ Having established that video ADs have a supplementary role to play in evidencing a person's expression of wishes, it is important to examine their legal and practical implications. The following analysis sets out the key implications arising from the use of video ADs.

\section{Legal and Practical Implications of Video ADs}

The option of video ADs invites us to reconsider wider issues and possibilities that may surface in the light of these developments. In this section I consider the legal and practical implications arising from the use of video ADs from the perspectives of validity, applicability, and authenticity. Recommendations on ways to address the implications are outlined thereafter.

\section{Validity}

The validity of an $\mathrm{AD}$ is determined from three aspects: mental capacity, voluntariness and an appreciation of the nature and consequences of such wishes to refuse treatment. ${ }^{58}$ Where there are doubts concerning any of the elements above, the decision-making process in ADs would be scrutinised further. Evidence of a person's capacity, voluntariness and understanding of the refusal or expression of treatment preferences are usually obtained from doctors, psychiatrists, consultants and the treatment team generally in order to confirm that the $\mathrm{AD}$ reflects the genuine

\footnotetext{
57 Moseley et al. (2005: 211, 213).

58 Cases have shown that ADs refusing treatment were declined on these grounds: $R e T$ [1992] EWCA Civ 18; NHS Trust v T (adult patient: refusal of medical treatment) [2004] EWHC 1279 (Fam); A Local Authority v E (by her Litigation Friend the Official Solicitor), A Health Authority \& E's Parents [2012] EWHC 1639 (COP).
} 
wishes of the person. ${ }^{59}$ Video ADs would also be subject to similar determinations. The question is whether it would be easier for video ADs, compared to written ADs in satisfying the demands of ascertaining the person's capacity, voluntariness and understanding of the information at the time they were made. Video ADs may be more self-explanatory in indicating signs of (in)capacity or other external circumstances as images are conveyed visually. Even if there was no opportunity to verify the mental capacity, voluntariness or understanding of the nature and consequences of the expressed wishes, the person's facial expressions, emotional state and composure revealed insights into the mental capacity and voluntariness at that particular time. To this end, video ADs may appear to meet the existing requirements more easily compared to written ADs. The context in which the video is made, including the timing, and who is around when the video is recorded are contributing factors in determining the validity of the video AD. As indicated above, one potential way to counter this layer of doubt is for the person to record the wishes in the presence of the doctor, with the person's consent, in an agreed setting.

\section{Applicability}

A valid AD needs to be applicable to the circumstances. ${ }^{60}$ As such, questions ranging from whether or not the decisions fall within the scope of the circumstances; changes in the personal or medical circumstances since the ADs were made or whether the person has had a change of mind often occur when ADs are sought to be applied. ${ }^{61}$ A written AD often suffers from this aspect, unless there is confirmation from external sources that the preferences remain or that it has been recently reviewed to reflect the most current wishes. Video ADs could equally risk vagueness especially where expressions such as "I do not wish to continue treatment if I suffer from intolerable pain" are made which would be subject to interpretations; or where the video was made weeks or months ago and there were doubts regarding the currency of the decision expressed in the video. ${ }^{62}$ In the case of the latter, video $\mathrm{ADs}$ are comparable to written $\mathrm{ADs}$ in terms of the time intervals between when

\footnotetext{
59 Examples from England and Wales, and Australia revealed the necessity of supplying proof of capacity when the validity of ADs is disputed. It is recommended that conversations and any capacity assessments are recorded and reduced to writing as evidence and patients are supported in the process of creating ADs: Chan (2018).

60 Section 25(1) Mental Capacity Act 2005 (UK).

61 Sections 25(2), (3), and (4) Mental Capacity Act 2005 (UK). The case of Re AK (Adult Patient)(Medical Treatment: Consent) [2001] 1 FLR 129 illustrated the firm decision of AK regarding treatment withdrawal two weeks after he had lost the ability to communicate with his eyes. The court was satisfied that the decision was current and reflected the view of AK. In contrast, the decision of HEv A Hospital NHS Trust \& AE (by her litigation friend the Official Solicitor) [2003] EWHC 1017 (Fam) demonstrated the change of personal circumstances resulting in the patient changing her mind regarding her refusal for blood transfusion.

62 A comparable example is the case of W Healthcare NHS Trust $v H$ and others [2004] EWCA Civ 1324 where there was no clear AD when the patient expressed that she would not want to be kept alive by machines and that she would not wish to be a burden to her daughters. The court found that these expressions were vague and made years ago, and did not constitute a sufficiently clear refusal.
} 
the decisions are sought to be implemented and when they are first created. There is similarly a need for patients to review the video ADs where there are changes, which would significantly affect the continued applicability of the AD, either on a monthly or half-yearly basis, depending on the particular circumstances of the patient.

Video ADs that are made privately may not incorporate information that is essential for doctors to apply them. One view is that a video AD should be as short and direct as possible to include important information such as the wishes, limits to treatment and choices of substitute decision-makers. ${ }^{63}$ Another possibility is for such videos to be recorded in the presence of doctors or nurse specialists, guided by crucial points that should be included in the narrative. This option would be beneficial for patients who may require support and guidance, both technical and medical information in deciding the content of the video AD and towards creating the video. A further implication is that recording video ADs could be time-consuming, and there may be limitations restricting doctors from participating in these sessions. An alternative would be for lawyers to assist in the process, such as witnessing the recording or verifying the sufficiency of information included in the recording.

What would be the implications for video ADs in emergencies when they are sought to be applied? This is a practical yet important consideration which would well occur in many clinical situations. There is virtually no time or not feasible to open the video file to interpret what the person has expressed in those critical moments. The process in recording patient wishes should equally consider this aspect and to take steps to implement ways to address this challenge.

\section{Authenticity}

The authenticity of video ADs is an important implication, as it raises both legal and ethical considerations. How authentic are these expressions of wishes projected through videos? The legal aspect of authenticity relates to questions of safety and security of the videos, which should be free from unauthorised interference and illegal access by unintended third party recipients. Where the videos are stored in the cloud, there is the likelihood of the recordings being compromised by online 'hackers' demanding ransoms for the release of personal information or cyber-attacks, which would put a dent in their credibility and reliability. This would necessitate anticipating ways to counter potential data breaches and security of video ADs recorded in digital formats. ${ }^{64}$ An approach would be agreeing on the level of access

\footnotetext{
63 Moseley et al. (2005: 211).

64 For example, some recent developments in Australia where a portal registry containing My Health Record is launched and is accessible at any time by patients and their healthcare providers: https:// www.myhealthrecord.gov.au/for-you-your-family/what-is-my-health-record. Accessed 10 January 2019. However there were concerns regarding privacy and confidentiality issues with the framework which is similar to the framework implemented in England and subsequently abolished when it was found to be in breach of patient privacy and confidentiality through sale of patient data: Zhou, 'My Health Record privacy framework 'identical' to failed UK scheme, expert says' (The Guardian, 21 July 2018) https:// www.theguardian.com/australia-news/2018/jul/22/my-health-record-identical-to-failed-uk-scheme-priva cy-expert-says?CMP=share_btn_fb. Accessed 10 January 2019.
} 
when these recordings were first created. If it was made in hospitals with doctors having a role in recording the wishes, they could be monitored within the hospital databases. Video recordings stored on third party platforms such as private hospitals, hospices, or care homes should equally apply rigorous security measures that protect the authenticity of the video ADs. An alternative suggestion is keeping the video ADs in "tamper-resistant sealed containers". ${ }^{65}$ In respect of the ethical consideration, it raises the question of how do we determine the authenticity of the wishes expressed in videos. This would be particularly relevant where minuscule emotional responses or distress displayed by the person narrating their wishes in the video may be erroneously assumed as exhibiting signs of incapacity or undue influences.

An ancillary but significant issue is the duplicity of recordings of video ADs. While it is generally accepted that the later recording is considered as the most recent expression of wishes that supersedes the earlier version(s), it becomes more complicated when there are several versions of the recordings found on multiple platforms. This could occur, for example, where patients have revoked their previous video $\mathrm{ADs}$ and replaced them with a newer version but had failed to notify anyone about the changes or taken steps to register the recording with the relevant parties, as the case may be. There are further questions regarding which version of the AD should prevail in the event of inconsistency between the video AD and any written AD that may exist at that time. How should doctors reconcile the multiplicity of video recordings? Should they accept the written AD over the video AD or vice versa? As proposed above, video ADs perform its supplementary role; thus, the written AD would be accepted as the prevailing document while any inconsistency between the provisions in the written $\mathrm{AD}$ and the video $\mathrm{AD}$ should be resolved in favour of the written AD, unless otherwise specified. Where this is intended as such, careful consideration needs to be put into writing the main AD document to avoid any future unintentional discrepancies. In terms of addressing the issue of multiplicity of video ADs, this point needs to be emphasised to the person who created the video that later versions of video ADs would be accepted as the last and most current wishes, and they would need to take steps to deal with this aspect if they did not intend it as such.

The implications identified above signalled that people should be careful in using video ADs. A written $\mathrm{AD}$ may be updated with a newer version or revoked, either by communicating the changes verbally, by destroying the previous $\mathrm{AD}$ or acting inconsistently with the ADs made previously. ${ }^{66} \mathrm{~A}$ video $\mathrm{AD}$ on the other hand may be implied as revoked by a later video recording, by an expression of revocation in words without necessarily recording a new video AD, or by acting in an inconsistent manner that signals the intention to depart from the AD.

\footnotetext{
65 Moseley et al. (2005: 211, 217).

66 Sections 24, 25(2) Mental Capacity Act 2005 (UK). See also NHS Trust v T (adult patient: refusal of medical treatment) [2004] EWHC 1279 (Fam) where the patient was persuaded to accept blood transfusion although she had reacted negatively to the idea initially.
} 


\section{Recommendations for Safeguards in Utilising Video ADs}

\section{Reporting and Access}

The introduction of video ADs requires a fresh set of response to address their accompanying challenges. One of the leading challenges in implementing ADs is timely access. ADs are often not found when they are needed the most. Reporting the existence of ADs would potentially reduce the risks of them being undiscovered, but this ought to be preceded by the patients notifying their doctors and next-of-kin of their existence. As such, once patients have recorded their ADs, they would need to report them to their doctors or notify their registered healthcare providers. Similarly, whenever advance care planning conversations occurred and ADs were created, they should be logged onto the hospital system along with the patient's medical records and notes. This should also apply to video ADs, whether they are made privately or within hospital settings. Crucially, the hospital infrastructure should be equipped with an integrated system where patient records can be accessed in a timely manner. This would mean either overhauling the current system or improving and upgrading connectivity and accessibility across the hospitals, which would necessitate allocating resources to fund their development and monitoring. There is consequently a need to prioritise this aspect in healthcare delivery, without which it would cost hospitals time and money. Information derived from these reporting would help towards devising strategies for implementing educational campaigns and raising awareness of video ADs.

An ancillary but important consideration is linking video ADs to clinical practice and addressing the issue of resource availability. Questions such as who should be responsible for these conversations; or should video ADs be recorded always in the presence of doctors are practical concerns relating to clinical time constraints, funding and availability of other resources. Competing healthcare demands may signify less urgent priority for making video ADs. The New Zealand experience showed that advance care planning training for healthcare professionals is effective in raising awareness and enabling more conversations in creating future care plans, such as providing feedback and reviewing completed plans, and uploading them into shared electronic records. Specialist nurses in advance care planning and allied health professionals have played a vital role in helping people engage in writing ADs.

\section{Reviewing and Updating Video ADs}

As with written ADs, video ADs need periodic reviews, and particularly necessary where circumstances have changed requiring different considerations. ${ }^{67}$ The issue here then is to what extent should the video recording be re-recorded when circumstances have changed? Material changes would imply that a fresh recording should be made, and good practice would necessitate a review where feasible—for example

67 The Mental Capacity Code of Practice recommended regular reviews and updating of ADs to ensure the currency of the decision: paragraphs 9.29 and 9.30 . 
every 6 months to ensure the currency of the wishes. Minor changes could be implemented via a transcript accompanying the video AD. More often than not, written ADs would be liable to be forgotten once they were made. Video ADs would also fall within the same category. One way to counter this would be for the hospital or any provider with whom the person has deposited and registered the ADs to send out notification texts or reminders to the person (containing a secure link via email or other forms of alerts) to review the video recording. This would operate in a similar way as a mobile notification or top-up alert. This approach is a small, yet significant step towards ensuring that people are reminded to review and update their ADs where necessary to ensure the continued applicability of their ADs.

\section{Developing a Registry for Video ADs}

The case of Brenda Grant (discussed above) demonstrated the need to establish a sound registry to store ADs. Hospitals need to institute better processes of communication to streamline the access of ADs in a timely manner. Developing a registry for video $\mathrm{ADs}$ is compatible with the increasing shift towards electronic management of patient records. The registry would contain relevant medical records, including their video ADs, with patients having access to the system. Some countries such as Armenia, Belgium and Germany already provide for registration systems within a central registry for written ADs. ${ }^{68}$ It is thus reasonable to infer the inclusion of video ADs as either part of the healthcare registration system or as a separate registry.

Parallel to this development, the legal framework needs to be revisited to accommodate the use of video ADs. For example, where there is a need to adhere to requirements in terms of signature, witnesses or using prescribed forms in the particular jurisdiction, questions would inevitably arise as to whether video ADs would be considered as acceptable forms of ADs. ${ }^{69}$ While electronic signatures may be able to meet the requirements of signatures akin to written ADs, video ADs may not sufficiently satisfy such requirements. In respect of witnessing the $\mathrm{AD}$, the video $\mathrm{AD}$ would require the presence or confirmation of witnesses, where possible. ${ }^{70}$ An option to address this implication is to include a statement in the recording to the effect that the video AD has been witnessed by a qualified person (most likely a doctor, lawyer or relevant allied healthcare professionals) or verified by the doctor. The formalities envisioned under the law as to the requirements of ADs would imply that the law is to be interpreted to include visual images where video ADs are accepted as conforming to prescribed forms or alternatively, adopting a favourable approach that includes video ADs as written ADs. ${ }^{71}$ For example, the Ireland

\footnotetext{
68 Ward (2018: 44).

${ }^{69}$ For example ss24 to 26 of the English Mental Capacity Act 2005 provides that an advance decision to refuse life sustaining treatment needs to be signed, witnessed and certified to the effect that the decision is to apply even at the risk of death.

${ }^{70}$ For instance where the AD relates to refusing life-sustaining treatment then the video AD would necessarily need to be witnessed accordingly.

71 Some European countries stipulate formal requirements that include writing and revoking ADs: Ward (2018: 30). This would imply that unless otherwise specified, video ADs would fall outside the remit of such formalities.
} 
Assisted Decision Making (Capacity) Act 2015 stipulates that an advance healthcare directive shall be in writing 72 and "writing" is interpreted to include "voice and video recording and speech recognition technologies",73 thus this option permits people to make video ADs.

\section{Conclusion}

This paper has sought to evaluate the position and role of video ADs within the ADs landscape. It examined the relevant legal implications arising from their use and proposed recommendations to address the challenges identified above. Video ADs offer the option of communicating medical treatment decisions through visual representations accompanied by voice recording. This development however raises essential legal questions in the framework governing healthcare decision-making. Questions regarding assessing the validity, applicability and authenticity of these video ADs raise important issues when they are called into implementation. Such a consideration is timely given the progressive shift towards electronic options of making and recording ADs. There are important implications for jurisdictions wishing to implement the law on ADs with the need to take into account these developments in the formulation of their law and the compatibility with current practices, particularly the ways in which the existing framework accommodates the challenges arising from video ADs.

While video ADs have several advantages over written ADs, they suffer from issues regarding the validity of the $\mathrm{AD}$ and the opportunity to review the decision, in addition to practical questions on storage, security and accessibility. Despite this weakness, they complement written ADs in their supplementary roles, as such there are opportunities to investigate other ways in which video ADs can assist advance care planning processes and consequently for hospitals to offer training and support to healthcare professionals in facing these concerns. Additionally, it is particularly significant to note that there is still a need to understand who the intended service users are and how video ADs can play an effective role in facilitating the implementation of their preferences. This includes broader questions regarding the awareness of the population in using video ADs, and familiarity with managing online registries or cloud storage.

Acknowledgements The author is grateful for the constructive feedback and comments received from the anonymous reviewers to the earlier drafts of this paper and to the participants of the $3^{\text {rd }}$ International Conference on End-of-Life Law, Ethics, Policy and Practice 2019 where this paper is presented.

\section{Compliance with Ethical Standards}

Conflict of interest The author declares that there is no conflict of interest.

\footnotetext{
72 Section 84(4) Assisted Decision Making (Capacity) Act 2015.

73 Section 82 Assisted Decision Making (Capacity) Act 2015.
} 
Open Access This article is distributed under the terms of the Creative Commons Attribution 4.0 International License (http://creativecommons.org/licenses/by/4.0/), which permits unrestricted use, distribution, and reproduction in any medium, provided you give appropriate credit to the original author(s) and the source, provide a link to the Creative Commons license, and indicate if changes were made.

\section{References}

A Local Authority v E (By Her Litigation Friend the Official Solicitor), A Health Authority \& E's Parents [2012] EWHC 1639 (COP).

Advance Care Plan. http://advancecareplan.org.uk/examples-document-templates/, http://advancecar eplan.org.uk/advance-care-plan-passport/. Accessed 5 January 2019.

Advance Care Directives Act 2013 (SA).

Advance Decisions Assistance. http://adassistance.org.uk/write-it/. Accessed 12 March 2019.

Advance Medical Directives Act $1996(\mathrm{Sg})$.

Advance Personal Planning Act 2013 (NT).

Advanced Health Care Directives Act 1995SNL c A-4.1.

An Act respecting end-of-life care Bill 2014 (52) c 2 (Quebec).

Assisted Decision Making (Capacity) Act 2015, s84(4).

Brauer, S., Biller-Andorno, N., and Andorno, R. 2008. The Country Reports on Advance Directives. European Science Foundation and the University of Zurich ESF Exploratory Workshop Advance Directives: Towards a Coordinated European Perspectives?

Brom, L., H. Roeline, W. Pasman, G.A.M. Widdershoven, M.J.D.L. van der Vorst, J.C. Reijneveld, T.J. Postma, and B.D. Onwuteaka-Philipsen. 2014. Patients' Preferences for Participation in Treatment Decision-Making at the End of Life: Qualitative Interviews with Advanced Cancer Patients. PLOS ONE 9 (6): e100435.

Buford, C. 2008. Advancing an Advance Directive Debate. Bioethics 22 (8): 423-430.

Chan, H.Y. 2018. Advance Directives: Rethinking Regulation, Autonomy and Healthcare DecisionMaking. Cham: Springer.

Compassion in Dying. https://compassionindying.org.uk/. Accessed 5 February 2019.

Consent to Treatment and Health Care Directives Act 1988 PEI c C-17.2.

Deep, K.S., A. Hunter, K. Murphy, and A. Volandes. 2010. "It Helps Me See With My Heart': How Video Informs Patients' Rationale for Decisions About Future Care in Advanced Dementia. Patient Education and Counseling 81 (2): 229-234.

Dresser, R. 1995. Dworkin on Dementia: Elegant Theory, Questionable Policy. Hastings Center Report 25 (6): 32-38.

Duckworth, S., and A. Thompson. 2017. Evaluation of the Advance Care Planning Programme. Wellington: Health Quality and Safety Commission.

Guardianship and Administration Act 1990 (WA).

Hanson, L.C., S. Zimmerman, M.-K. Song, et al. 2017. Effect of the Goals of Care Intervention for Advanced Dementia: A Randomized Clinical Trial. JAMA Internal Medicine 177 (1): 24-31.

HE v A Hospital NHS Trust \& AE (By Her Litigation Friend the Official Solicitor) [2003] EWHC 1017 (Fam).

Health Care (Consent) and Care Facility (Admission) Act RSBC 1996 c 181.

Health Care Consent Act 1996 SO c2.

Health Care Directives Act 1992 CCSM c H27.

Health Care Directives and Substitute Health Care Decision Makers Act 1997 S c H-0.001.

Horn, R.J. 2014. Advance Directives in English and French Law: Different Concepts, Different Values, Different Societies. Health Care Analysis 22 (1): 59-72.

http://www.health.wa.gov.au/docreg/education/population/HP11536_advance_health_directive_form. pdf. Accessed 10 March 2019.

http://www.npmc.nhs.uk/website/K82016/files/Living\%20will\%20Forms\%20for\%20patients\%20upd ate\%202018.pdf. Accessed 14 March 2019.

https://advancecaredirectives.sa.gov.au/forms-and-guides. Accessed 10 March 2019.

https://mydecisions.org.uk/?utm_source=cidwebsite\&utm_medium=planningahead\&utm_campa ign=CIDPLANAHEAD. Accessed 5 January 2019. 
https://www2.health.vic.gov.au/hospitals-and-health-services/patient-care/end-of-life-care/advancecare-planning/acp-forms. Accessed 14 March 2019.

https://www.aarp.org/caregiving/financial-legal/free-printable-advance-directives/. Accessed 14 March 2019.

https://www.advancecareplanning.org.au/individuals/advance-care-directives-and-the-law. Accessed 10 March 2019.

https://www.health.nsw.gov.au/patients/acp/Publications/acd-form-info-book.pdf. Accessed 10 March 2019.

https://www.mydeathmydecision.org.uk/hospital-trust-pays-45000-ignoring-advance-decision/. Accessed 14 March 2019.

https://www.myhealthrecord.gov.au/for-you-your-family/what-is-my-health-record. Accessed 10 January 2019.

In My Own Words. http://inmyownwords.com/. Accessed 5 February 2019.

Independent Age. Older People, Technology And Community: The Potential Of Technology to Help Older People Renew or Develop Social Contacts and to Actively Engage in Their Communities. https://www. cisco.com/c/dam/en_us/about/ac79/docs/wp/ps/Report.pdf. Accessed 2 January 2019.

Jain, A., S. Corriveau, K. Quinn, A. Gardhouse, D.B. Vegas, and J.J. You. 2015. Video Decision Aids to Assist with Advance Care Planning: A Systematic Review and Meta-analysis. British Medical Journal Open 5: e007491.

Klugman, C.M., and R.P. Usatine. 2012. An Evaluation of 2 Online Advance Directive Programs. American Journal of Hospice \& Palliative Medicine 30 (7): 657-663.

Maclean, A.R. 2006. Advance Directives, Future Selves and Decision-Making. Medical Law Review 14 (3): 291-320.

Maclean, A.R. 2008. Advance Directives and the Rocky Waters of Anticipatory Decision-Making. Medical Law Review 16 (1): 1-22.

Macmillan Cancer Support Advance Decision to Refuse Treatment. https:/www.macmillan.org.uk/_images/ ADRTDocument_20170505_JS_tcm9-311758.pdf. Accessed 5 January 2019.

Marso, Andy. Last Wishes: Use Video to Tell Doctors and Family What You Want at Death's Door' (Kansas City Star, 21 April 2017). https://www.kansascity.com/news/business/health-care/article14562569 9.html. Accessed 2 February 2019.

Medical Treatment (Health Directions) Act 2006 (ACT).

Medical Treatment Planning and Decisions Act 2016 (Victoria, Australia).

Mental Capacity Act 2005 (UK), s 24.

Mirarchi, F.L., et al. 2017. TRIAD VIII: Nationwide Multicenter Evaluation to Determine Whether Patient Video Testimonials Can Safely Help Ensure Appropriate Critical versus End-of-Life Care. Journal of Patient Safety 13 (2): 51-61.

Mitchell, M. 2012. An Analysis of Common Arguments Against Advance Directives. Nursing Ethics 19 (2): 245-251.

Mitchell, S.L., M.L. Shaffer, S. Cohen, L.C. Hanson, D. Habtemariam, and A.E. Volandes. 2018. An Advance Care Planning Video Decision Support Tool for Nursing Home Residents With Advanced Dementia: A Cluster Randomized Clinical Trial. JAMA Internal Medicine 178 (7): 961-969.

Moseley, R., A. Dobalian, and R. Hatch. 2005. The Problem with Advance Directives: Maybe it is the Medium, Not the Message. Archives of Gerontology and Geriatrics 41: 211-219.

My Decisions. https://mydecisions.org.uk/. Accessed 12 March 2019.

My Directives. https://mydirectives.com/. Accessed 5 February 2019.

NHS Trust v T (Adult Patient: Refusal of Medical Treatment) [2004] EWHC 1279 (Fam).

Ofcom. Adults' Media Use and Attitudes Report. 25 April 2018.

Olick, R.S. 2012. Defining Features of Advance Directives in Law and Clinical Practice. Chest 141 (1): 232-238.

Paduano, M. Payout After Woman was Kept Alive Against Her Will' (BBC, 6 December 2017). https://www. bbc.co.uk/news/uk-england-coventry-warwickshire-42240148. Accessed 14 March 2019.

People in the EU: Statistics on an Ageing Society (Eurostat, 7 January 2019). https://ec.europa.eu/eurostat/ statistics-explained/index.php/People_in_the_EU_-_statistics_on_an_ageing_society. Accessed 9 January 2019.

Personal Directives Act 2000 RSA c P-6.

Personal Directives Act 2005 SNWT c 16. 
Personal Directives Act 2008 SNSc 8.

Phil Cheatle. MDMD. Hospital trust pays out $£ 45,000$ for ignoring an Advance Decision (My Death My Decision, 6 December 2017).

Powers of Attorney Act 1998 (Qld).

Re AK (Adult Patient)(Medical Treatment: Consent) [2001] 1 FLR 129.

Re C (refusal of medical treatment) [1994] 1 WLR 290 (Fam).

$\operatorname{Re} D$ [2012] EWHC 885 (COP).

Re T [1992] EWCA Civ 18.

Rise of the Social Seniors Revealed (Ofcom, 14 June 2017). https://www.ofcom.org.uk/about-ofcom/latest/ media/media-releases/2017/rise-social-seniors. Accessed 9 January 2019.

Ruseckaite, R., K.M. Detering, and S.M. Evans. 2017. Protocol for a National Prevalence Study of Advance Care Planning Documentation and Self-reported Uptake in Australia. British Medical Journal Open 7: $\mathrm{e} 018024$.

Ryan, C.J. 1996. Betting Your Life: An Argument against Certain Advance Directives. Journal of Medical Ethics 22 (2): 95-99.

Sandza, R. 2017. Video Advance Directives: Your Smartphone Records Your Wishes. Bifocal 38(5). https:// www.americanbar.org/publications/bifocal/vol_38/issue-5-june-2017-/video-advance-directives-yoursmartphone-records-your-wishes.html. Accessed 5 January 2019.

Say, R.E., and R. Thomson. 2003. The Importance Of Patient Preferences in Treatment Decisions-Challenges for Doctors. BMJ 327: 542-545.

Sepucha, K., and A.G. Mulley. 2009. A Perspective on the Patient's Role in Treatment Decisions. Medical Care Research and Review 66 (1): 53S-74S.

Silveira, M.J., S.Y. Kim, and K.M. Langa. 2010. Advance Directives and Outcomes of Surrogate DecisionMaking Before Death. New England Journal of Medicine 362: 1211-1218.

Silveira, M.J., W. Wiitala, and J. Piette. 2014. Advance Directive Completion by Elderly Americans: A Decade of Change. Journal of the American Geriatrics Society 62 (4): 706-710.

Simón, P. 2011. Use of Advance Care Planning-A European Perspective. BMJ Supportive \& Palliative Care 1: 75.

Sudore, R.L., S.J. Knight, R.D. McMahan, M. Feuz, D. Farrell, Y. Miao, and D.E. Barnes. 2014. A Novel Website to Prepare Diverse Older Adults for Decision Making and Advance Care Planning: A Pilot Study. Journal of Pain and Symptom Management 47 (4): 674-686.

Toraya, C. 2014. Evaluation of Advance Directives Video Education for Patients. Journal of Palliative Medicine 17 (8): 942-946.

Umar, N., D. Litaker, M.-L. Schaarschmidt, W.K. Peitsch, A. Schmieder, and D.D. Terris. 2012. Outcomes Associated with Matching Patients' Treatment Preferences to Physicians' Recommendations: Study Methodology. BMC Health Services Research 12: 1-10.

Vearrier, L. 2016. Failure of the Current Advance Care Planning Paradigm: Advocating for a Communications-Based Approach. HEC Forum 28 (4): 339-354.

Veshi, D., and G. Neitzke. 2015. Advance Directives in Some Western European Countries: A Legal and Ethical Comparison between Spain, France, England, and Germany. European Journal of Health Law 22 (4): 321-345.

Volandes, A.E., M.K. Paasche-Orlow, M.J. Barry, M.R. Gillick, K.L. Minaker, Y. Chang, E.F. Cook, E.D. Abbo, A. El-Jawahri, and S.L. Mitchell. 2009. Video Decision Support Tool for Advance Care Planning in Dementia: Randomised Controlled Trial. BMJ 338: b2159.

Volhard, T., F. Jessen, L. Kleineidam, S. Wolfsgruber, D. Lanzerath, M. Wagner, et al. 2018. Advance Directives for Future Dementia can be Modified by a Brief Video Presentation on Dementia Care: An Experimental Study. PLoS ONE 13 (5): e0197229.

W Healthcare NHS Trust $v$ H and Others [2004] EWCA Civ 1324.

Ward, AD. 2018. Enabling Citizens to Plan for Incapacity: A Review of Follow-Up Action Taken by Member States of the Council of Europe to Recommendation CM/Rec(2009)11 on Principles Concerning Continuing Powers of Attorney and Advance Directives for Incapacity. European Committee on Legal Co-operation (CDCJ) Council of Europe.

Who decides? Making Decisions on Behalf of Mentally Incapacitated Adults: A Consultation Paper issued by the Lord Chancellor's Department Presented to Parliament by the Lord High Chancellor by Command of Her Majesty December 1997 (Cm 3803). 
Wilson, M.E., A. Krupa, R.F. Hinds, J.M. Litell, K.M. Swetz, A. Akhoundi, R. Kashyap, O. Gajic, and K. Kashani. 2015. A Video to Improve Patient and Surrogate Understanding of Cardiopulmonary Resuscitation Choices in the ICU: A Randomized Controlled Trial. Critical Care Medicine 43 (3): 621-629.

Zhou, Naaman. My Health Record Privacy Framework 'Identical' to Failed UK Scheme, Expert Says (The Guardian, 21 July 2018). https://www.theguardian.com/australia-news/2018/jul/22/my-health-recor d-identical-to-failed-uk-scheme-privacy-expert-says?CMP=share_btn_fb. Accessed 10 January 2019.

Publisher's Note Springer Nature remains neutral with regard to jurisdictional claims in published maps and institutional affiliations. 